\title{
Three-Dimensional Vorticity Dynamics in a Coflowing Turbulent Jet Subjected to Axial and Azimuthal Perturbations
}

\author{
Katherine Prestridge and Juan C. Lasheras \\ Department of Applied Mechanics and Engineering Sciences \\ University of California, San Diego \\ La Jolla, CA 92093-0411, USA
}

\begin{abstract}
The effect of axial and azimuthal forcing on the spreading and mixing characteristics in the near field $(x / D<13)$ of a coflowing, turbulent $(R e>2000)$ water jet is studied by means of planar, laser induced fluorescence (PLIF) visualizations. Measurements of both the initial spreading rate of the jet and the decay of the concentration along the centerline of the jet show a marked increase in the mixing of the jet as a result of the axial forcing. The mixing enhancement, which is shown to depend on the wavenumber of the azimuthal forcing, the frequency of the axial forcing, and the Reynolds number of the jet, results from the formation of vortex loops caused by the global induction of the jet's vorticity. This vortex induction mechanism appears to be similar to the vorticity modes earlier found in laminar coflowing jets (Lasheras et al., Lect. Appl. Math., 28, 1991).
\end{abstract}

\section{Introduction}

Axisymmetric turbulent jets occur in a variety of applications: Combustion and heat transfer processes, gas-liquid separators, and jet engine exhausts. Depending upon the application, an enhancement in the mixing and entrainment of the jet in the near field, as well as far downstream, could improve the performance characteristics of the processes. Necessary to any control of the evolution of turbulent jets is an understanding of the underlying vorticity patterns that develop both naturally and when the jet is perturbed.

Under certain conditions: Reynolds number, density ratio, and temperature ratio, turbulent jets are known to develop side jets, which result in an enhancement in the spreading and mixing of the jet $[10,11,12]$. In previous studies of heated and variable density jets, lateral ejections have been observed emanating from the jet in the radial direction. In hot-air jet experiments, Monkewitz et al. [10] observed side jets that were "mainly ejected from the core or the immediate neighborhood of the primary vortex rings." The density ratio of the jet fluid to the ambient fluid, $S$, is important to the behavior of the jet, and in the hot-air jet experiments, the dominant Strouhal number differs as a function of this density ratio. For $S<0.7$, the jet becomes self-excited due to the absolute instability in the potential core. Although several mechanisms have been proposed to explain this behavior, to date there is no clear consensus as to the mechanisms responsible for this anomalous spreading.

Recent experiments with laminar coflowing jets subjected to the combined effect of axial and azimuthal forcing $[6,7,9]$ have shown that under certain forcing conditions the jets exhibit a variety of entrainment patterns. Some of these patterns closely resemble the side jet patterns 
observed in the turbulent case. These observations lead to several questions concerning turbulent jets, primarily: Are the mechanisms observed in the laminar jets the same ones responsible for the lateral ejections observed in turbulent jets? Can our understanding of the vortex dynamics involved in the forced laminar jets be extended to forced turbulent jets, where additional effects produced by the smaller scales may play an important role in the evolution of the flowfield?

In order to begin to answer these questions, we examine the effects of axial and azimuthal forcing on a turbulent water jet. The purpose of the axial forcing is to recreate the absolute instability of the variable density jets by locking the convective instability. An explanation of the experimental observations of the laminar jets will outline the motivation for the methodology used in this experiment, so before proceeding, we will highlight the important observations of the laminar coflowing jet experiments.

\section{Laminar jet study}

The experimental observations and numerical simulations of axially and azimuthally perturbed jets show that these perturbations evolve downstream into vortex rings and vortex tubes. The spanwise vortex rings are affected by the evolution of counter-rotating pairs of vortex tubes along the streamwise plane of strain. The interaction of these structures is dependent upon the initial conditions: number of corrugations in the azimuthal direction, and frequency and amplitude of the axial perturbation. The interaction between the regions of vorticity can be explained by inviscid vortex dynamics, both in the case of planar free shear layers [3, 4, 5], plane wakes [8], and jets $[6,7,9]$.

In the experimental study of laminar coflowing jets which are axially and azimuthally forced, [6], the existence of a large variety of entrainment patterns was found resulting from different modes of induction of the vorticity of the jet. One of these modes of behavior is pictured in Fig. 2, an image of the centerline of a 5-lobed, laminar jet. Due to the combined effect of axial and azimuthal forcing, the instability of the vortex rings formed in the jet results in the formation of loops of vorticity. There are several modes of behavior which result from the instability of the vortex rings emanating from the jet, and the mixing and entrainment patterns exhibited by the laminar forced jets are the primary motivation for the present study. Although a complete characterization of the behavior of the jet was not developed, there were several modes of three-dimensional vorticity dynamics which could be discerned using flow visualization techniques. The results for a Reynolds number of 1000 based on the inner jet diameter and the difference in velocity between the jet and coflow will be presented. The observations of the behavior of the 5-lobed nozzle provide an excellent example of the characteristic jet behavior. The 5-lobed corrugated nozzle was used to create a static perturbation in the azimuthal direction, and the axial forcing was created using a speaker diaphragm with a forcing frequency ranging between 4 and $20 \mathrm{~Hz}$.

The first mode of behavior occurs for small amplitude forcing and forcing frequencies which result in wavelengths on the order of the jet diameter, as pictured in Fig. 1. The cylindrical layer of vorticity rolls up into a periodic array of vortex rings. Counter-rotating pairs of streamwise vortices connect the vortex rings, which exhibit a wavy pattern corresponding to the corrugations of the nozzle. Numerical investigations using vortex filaments [7] agree very well with the experimental observations of the first mode of behavior.

As the axial forcing frequency is increased to that near the most unstable frequency, a second mode develops, as pictured in Fig. 2. If we examine a plane located three jet-diameters downstream and parallel to the exit plane of the nozzle, a pattern of lateral ejections is observed emanating from the jet centerline, Fig. 3 . This pattern is caused by the formation of a pair of vortex rings of opposite 
sign. The rings consist of a stronger inner ring, with waviness aligned with the corrugations of the nozzle, and a weaker outer ring, which is staggered with respect to the inner ring. The vorticity induction of these two rings causes the outer ring to further deform until loops of vorticity are formed through a process of vortex reconnection and pinching off. These loops are then selfpropelled laterally away from the jet centerline under their own induction.

As the axial forcing frequency is increased further, a third mode appears, Fig. 4, and there are closed vortex loops which are ejected laterally from the jet at positions corresponding to the peaks of the corrugations of the nozzles. This leads to a dramatic increase in the lateral spreading of the jet. The final mode observed in the jet is after another increase of the axial forcing frequency, where the number of lateral ejections is now double that of the previous modes. So, the 5-lobed nozzle produces a vortex ring structure with ten waves in the azimuthal direction, and this fourth mode produces an increase in the surface area of the jet-coflow interface. The discovery of these modes has prompted us to examine the turbulent jet for similar behavior in the hopes of improving the performance and understanding of these jets in their many technological applications.

\section{Experimental facility and observation techniques}

The experimental facility consists of a horizontally oriented water channel, Fig. 5. the channel flow is maintained at a uniform, steady velocity of $10.8 \mathrm{~cm} / \mathrm{s}$, in the laminar regime. The jet is supplied independently using a gravity feed system at constant head in order to avoid acoustic contamination of the jet flow by the pumps. The mean jet velocity ranges from $20 \mathrm{~cm} / \mathrm{s}$ to $50 \mathrm{~cm} / \mathrm{s}$, and the average jet diameter is $D_{j}=2.8 \mathrm{~cm}$. The flow parameters are nondimensionalized using the difference in velocity between the jet and coflow, $\Delta V$. With the Reynolds number defined as $R e=D_{j} \Delta V / \nu$, the resulting range is $2000<R e<30000$.

The results reported here correspond to the cases of a combined static perturbation in the azimuthal direction and dynamic perturbation in the axial direction. The azimuthal perturbation is introduced through the use of corrugated nozzles, with a corrugation amplitude of $0.1 D_{j}$, and the number of corrugations, $N$, varied between four and eight. The axial perturbation is introduced using a sinusoidally driven plunger mechanism far upstream of the jet exit (Fig. 5). The amplitude of the axial perturbation is always kept large, $(>10 \%)$. The axial forcing frequency, $f$, is varied up to $7 \mathrm{~Hz}$, and is referred to in terms of a nondimensional Strouhal number, $S t=f D_{j} / \Delta V$, where $0<S t<1.5$.

Fluorescein dye is introduced into the jet stream for the purpose of visualizing the spreading of the jet fluid into the outer coflowing stream. An Argon-ion laser sheet is projected into the test section, Fig. 6, using a high-speed, rotating mirror. For the present study, the images under analysis are confined to vertical, centerline images, which illuminate the fluorescein particles in a region spanning from the nozzle exit to thirteen jet diameters downstream, $0<x / D_{j}<13$. Visualization of the centerline is recorded using a digital (CCD) camera, where the light intensity of the excited fluorescein registers the concentration of jet fluid at each downstream location in the plane of illumination.

In order to obtain a first global quantification of the mixing and entrainment characteristics of the flow, we are initially examining only mean flow quantities. The time-averaged images are created using 32-frame averaging of the digitized images taken from the CCD camera at 30 frames per second. From these time-averaged images, we define parameters which will quantify the mean spreading characteristics of the jet. One of these measurements will be of the mean concentration along the centerline of the jet, which will be used to determine the average amount of jet fluid being ejected laterally away from the centerline. Another measure of the amount of lateral spreading will 
be visualizations of the level thicknesses of concentration of jet fluid, normalized with the centerline (maximum) value, as a function of axial forcing frequency. These images will give us some idea of the lateral "spreading angles" of the forced jets.

There are many ways to quantify the three-dimensional mixing characteristics of the jet. In order to determine the mean spreading of the jet fluid, we will also examine the mean normalized intensity of the half-jet, defined as:

$$
\bar{I}(r, x)=\frac{1}{(x / D)_{\max }} \int_{x=0}^{(x / D)_{\max }} \frac{1}{r(x)} \int_{r=0}^{r_{1 \% \max }} \frac{I}{I_{\max }} d r d \frac{x}{D}
$$

This is the mean value of the normalized fluorescein concentration between the centerline and edge of the jet as defined by the location of the $1 \% I_{\max }$ level thickness. This is measured by the light intensity captured by the CCD camera. After normalization with the integration length, which for all results presented is $(x / D)_{\max }=13$, and the local radius, $r(x), \bar{I}(r, x)$ varies between 0 and 1. The maximum intensity along the centerline, $I_{\max }$, occurs in the potential core and is used to normalize the intensity along the centerline. The mean normalized intensity of the half-jet, $\bar{I}$, provides us with a measure of the mixedness of the time-averaged jet concentration profile in the downstream direction. A decrease in $\bar{I}$ signifies that more jet fluid is being moved away from the centerline, and subsequently, more of the coflowing fluid is being entrained by the jet.

\section{Results and discussion}

Initially we analyze the effect of the static azimuthal perturbation on the jet in the case of no axial forcing. As we vary the wavenumber of the corrugated nozzles, it is immediately clear from Fig. 7 that the corrugated nozzles have enhanced mixing properties over the round nozzle for a fixed Reynolds number of 2500.

The enhanced mixing manifests itself as a more rapid drop in the mean centerline concentration. However, the effect of the number of lobes is not monotonic, possibly showing the existence of an optimum number of corrugations, which may vary with Reynolds number. The length of the potential core based on the centerline concentration remains relatively unaffected by the corrugations, staying at $x / D=3$ for all of the cases at this Reynolds number. These results compare qualitatively with the effects of tabs on the decay of the jet centerline velocity observed by Bradbury and Khadem [1]. Using the axisymmetric positioning of tabs inside the jet in order to produce an azimuthal perturbation of the jet, they observe the fastest decay of the centerline velocity in the case of two tabs, with the eight-tabbed jet behaving most similary to the round and having a slower rate of decay along the centerline [1].

Two centerline velocity profiles similar to those of the round and 8-lobed nozzles in Fig. 7 were observed for the case of a round jet which was axially forced at $S t=0.3$ [2]. In this classic study of the coherent structures in jet turbulence, Crow and Champagne [2] saw an increase in the entrainment of ambient fluid toward the jet centerline when they introduced axial forcing. There seemed to be a natural frequency for peak resonance of the jet and the formation of coherent structures at $S t=0.3$. The amplitude of their forcing was at $2 \%$ of the mean exit velocity of the jet, however, and we estimate that the amplitude of the forcing in this experiment is an order of magnitude larger than that. For this reason, it is important to examine a wide range of Reynolds numbers, as well as axial forcing frequencies which result in $S t>0.3$, in order to characterize the behavior of the jet.

The addition of an axial perturbation to the jet results in a dramatic effect on the near-field mixing. The addition of high-amplitude axial forcing to the jet flow creates highly reproducible 
structures at the jet exit, which are pictured in instantaneous images of the centerline of the 4-lobe jet shown in Fig. 8. These large structures resemble thick rings of vorticity for up to $x / D=5$, and the rings lose their initial coherence as they propagate further downstream. In this process, several key features are visible, including the spreading of the initial vortex blob, and the lateral ejection of smaller pieces of fluid from these structures as they move downstream. These lateral ejections are qualitatively similar to those found in variable density jets as well as the laminar jet studies, and they explain the dramatic increase in the lateral spreading rates. This increase in spreading corresponds to a decrease in $\bar{I}$, the mean normalized intensity of the jet.

We can compare the effects of axial forcing on all of the nozzles at a fixed Reynolds number by examining the change in $\bar{I}$. Figure 9 shows that there is an overall decrease in the mixing parameter as the forcing frequency is increased. At this Reynolds number, the round and 8-lobed nozzles behave similarly, as do the 5- and 7-lobed nozzles, while the 4-and 6-lobed nozzles realize the largest increase in mixing over the range of Strouhal numbers presented. The effects of the corrugated nozzles on the mixing of the jet are not monotonic, as illustrated again in Fig. 9, with the optimal mode being some combination of number of lobes and axial forcing frequency over a range of Reynolds numbers. The decrease in the value of $\bar{I}$ serves to quantify the dramatic increase in the mean mixing rate as the axial forcing is applied. Fig. 9 shows a $40 \%$ drop in $\bar{I}$ at $S t=1.3$ when compared to the unforced case. At $R e=2500$, the smallest decrease in mixing enhancement caused by the axial forcing occurred in the 5- and 7-lobed nozzles, where $\bar{I}$ still decreased by $20 \%$ at the higher forcing frequencies.

The movement of jet fluid away from the centerline is illustrated in Fig. 10, where the concentrations of fluid along the centerlines of the round and 4-lobes nozzles are compared at at fixed Reynolds number. The addition of axial forcing decreases the length of the potential core, while causing an increase in the lateral ejection of jet fluid away from the centerline. There also appear to be two distinct rates at which the concentration drops for the Strouhal numbers over 0.5: the steeper drop in concentration occurring at $x / D<5$, with the concentration profile dropping less steeply for $x / D>5$.

The increase in lateral spreading caused by the axial forcing is illustrated for the round jet in Fig. 11(a), where the level thicknesses which are $10 \%$ of the centerline concentration are shown. The location of the nozzle is also pictured for clarity. At a fixed Reynolds number of 2000, the increase in lateral spreading due to the increasing Strouhal number is small. When azimuthal corrugations are added, as in the case of the 4-lobed nozzle, Fig. 11(b), the increased lateral spreading of the jet at the same axial forcing frequencies is visible in the near field.

The spreading of the jets laterally compares well with the observations in hot jets [10], where half-angles of up to $45^{\circ}$ were observed. The similar resulting phenomena between the strongly forced jets and the heated jets lends support to the idea that vortex dynamics found in the laminar case and corroborated to exist in the turbulent forced jets could be responsible for the behavior reported in the heated jets.

\section{Conclusions}

Preliminary results of PLIF visualizations show that the near field entrainment and mixing patterns of a turbulent jet can be modified through the use of a combination of axial forcing and static azimuthal perturbations. It was shown that the mechanism responsible for this mixing modification appears to be qualitatively similar to the one found in laminar coflowing jets. However, it still remains to be further quantified whether or not the turbulent jets exhibit the same variety of vortex dynamics leading to the different entrainment modes found in the laminar case. Furthermore, 
although the current results show the possible existence of an optimum combination of Strouhal

number (axial forcing frequency), Reynolds number, and number of lobes, the optimum parameter combination remains to be found.

\section{References}

[1] Bradbury, L.J.S. and Khadem, A.H., "The Distortion of a Jet by Tabs," J. Fluid Mech., 70, pp. 801-813, 1975.

[2] Crow, S.C. and Champagne, F.H., "Orderly Structure in Jet Turbulence," J. Fluid Mech., 48, pp. 547-591, 1971.

[3] Lasheras, J.C., Cho, J.S. and Maxworthy, T., "On the Origin and Evolution of Streamwise Vortical Structure in a Plane, Free Shear Layer," J. Fluid Mech., 172, pp. 231-258, 1986.

[4] Lasheras, J.C. and Choi, H., "Three-Dimensional Instability of a Plane Free Shear Layer: An Experimental Study of the Formation and Evolution of Streamwise Vortices," J. Fluid Mech., 189, pp. 53-86, 1988.

[5] Lasheras, J.C. and Meiburg, E., "Three-Dimensional Vorticity Modes in the Wake of a Flat Plate," Phys. Fluids A, 2, pp. 371-380, 1990.

[6] Lasheras, J.C., Lecuona, A. and Rodriguez, P., "Three-Dimensional Vorticity Dynamics in the Near Field of Coflowing Forced Jets," Lect. Appl. Math., 28, pp. 403-422, 1991.

[7] Martin, J.E. and Meiburg, E., "Numerical Investigation of Three-Dimensionally Evolving Jets subject to Axisymmetric and Azimuthal Perturbations," J. Fluid Mech., 230, pp. 271-318, 1991.

[8] Meiburg, E. and Lasheras, J., "Experimental and Numerical Investigation of the ThreeDimensional Transition in Plane Wakes," J. Fluid Mech., 190, pp. 1-37, 1988.

[9] Meiburg, E., Lasheras, J.C. and Martin, J.E., Turbulent Shear Flows 7, Springer-Verlag, Berlin, 1991.

[10] Monkewitz, P.A., Bechert, D.W., Barsikow, B. and Lehmann, B., "Self-Excited Oscillations and Mixing in a Heated Round Jet," J. Fluid Mech., 213, pp. 611-639, 1990.

[11] Monkewitz, P.A., Lehmann, B., Barsikow, B. and Bechert, D.W., "The Spreading of SelfExcited Hot Jets by Side-Jets," Phys. Fluids A, 1, pp. 446-447, 1989.

[12] Sreenivasan, K.R., Raghu, S. and Kyle, D., "Absolute Instability in Variable Density Round Jets," Exp. in Fluids, 7, pp. 309-317, 1989. 


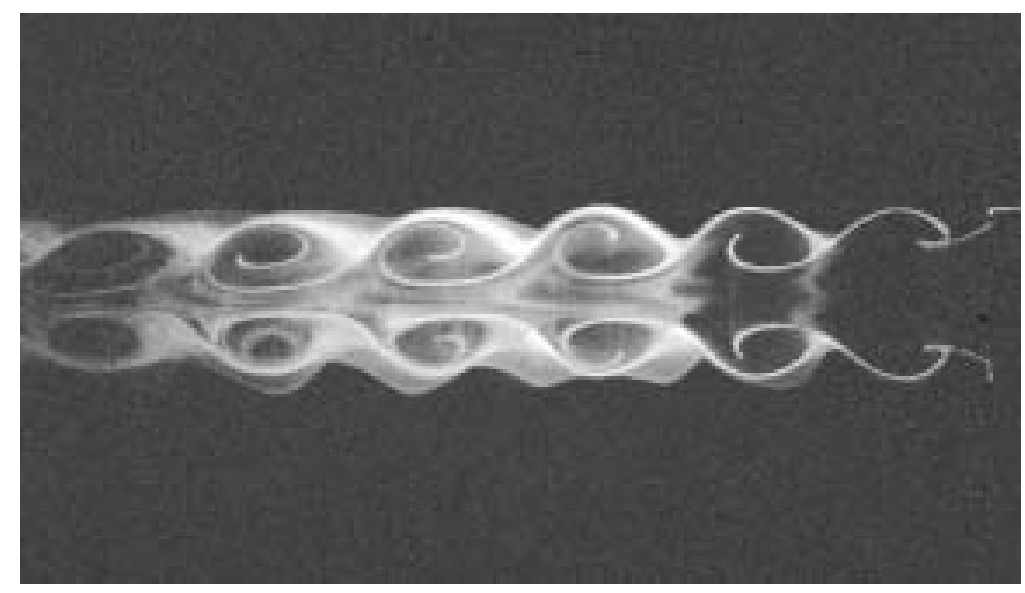

Figure 1: Centerline image of the forced laminar jet: Mode 1, from [6].

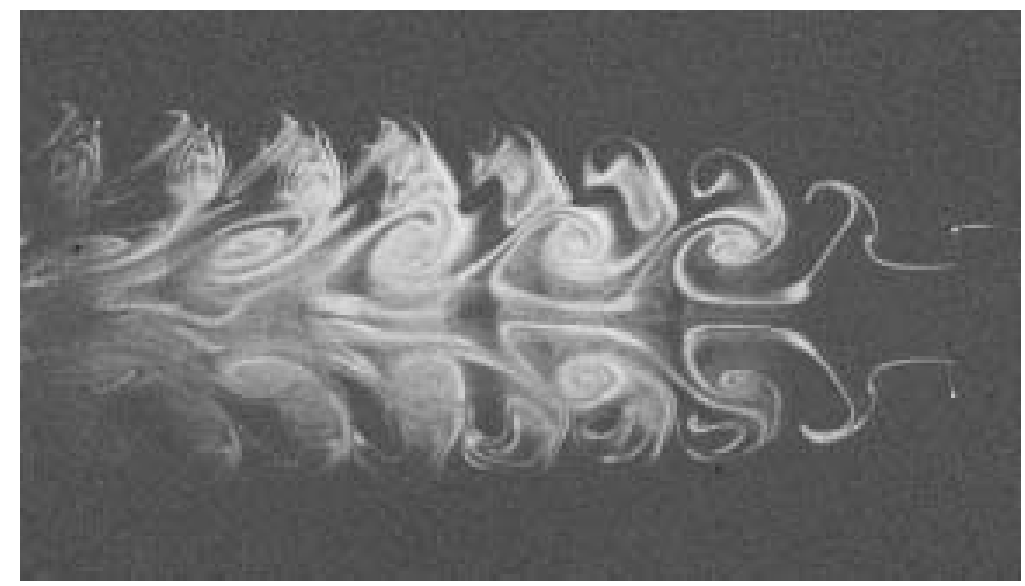

Figure 2: Centerline image of the forced laminar jet: Mode 2, from [6].

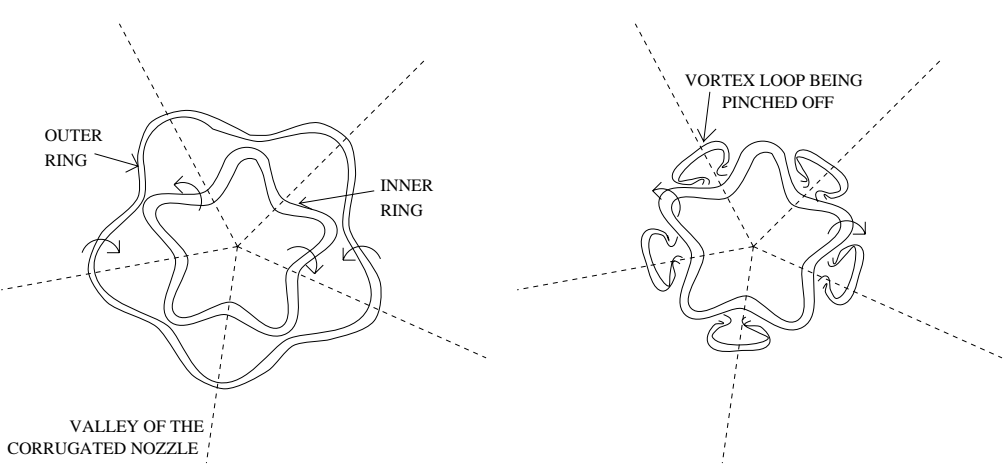

Figure 3: Mode two of the forced laminar jet, from [6]. 


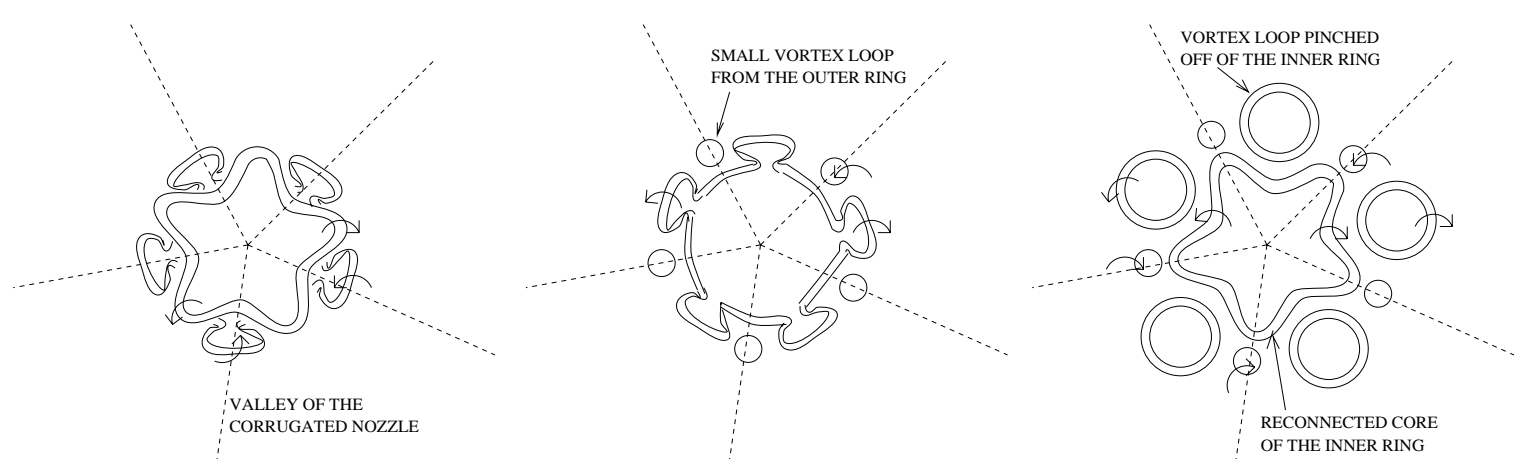

Figure 4: Mode three of the forced laminar jet, from [6].

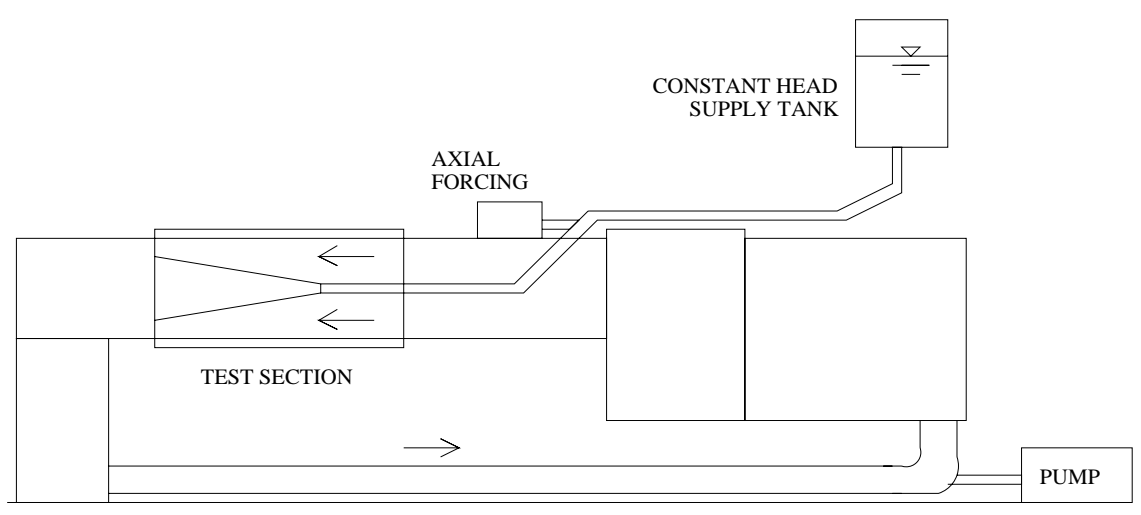

Figure 5: Experimental facility.

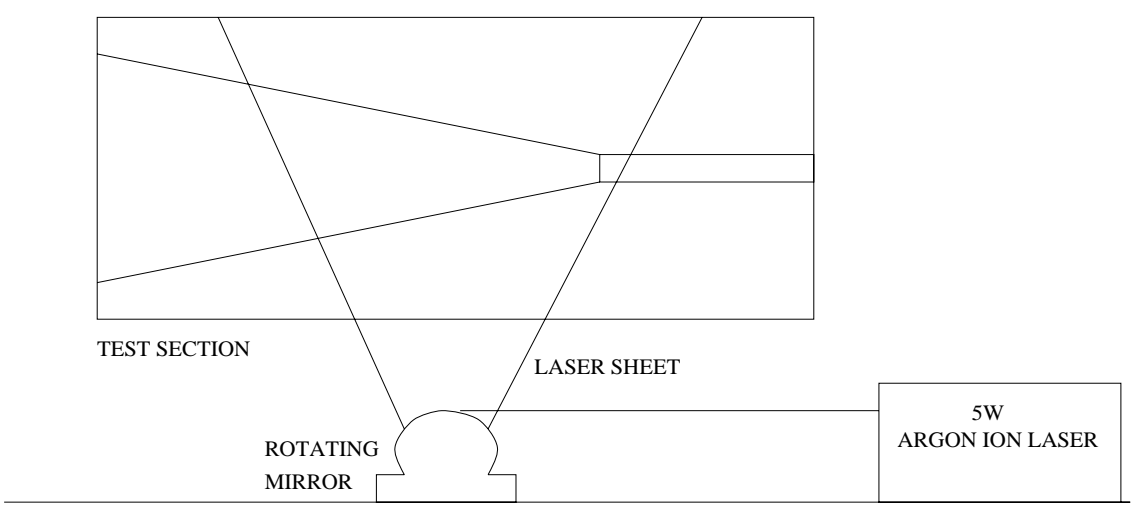

Figure 6: Test section. 


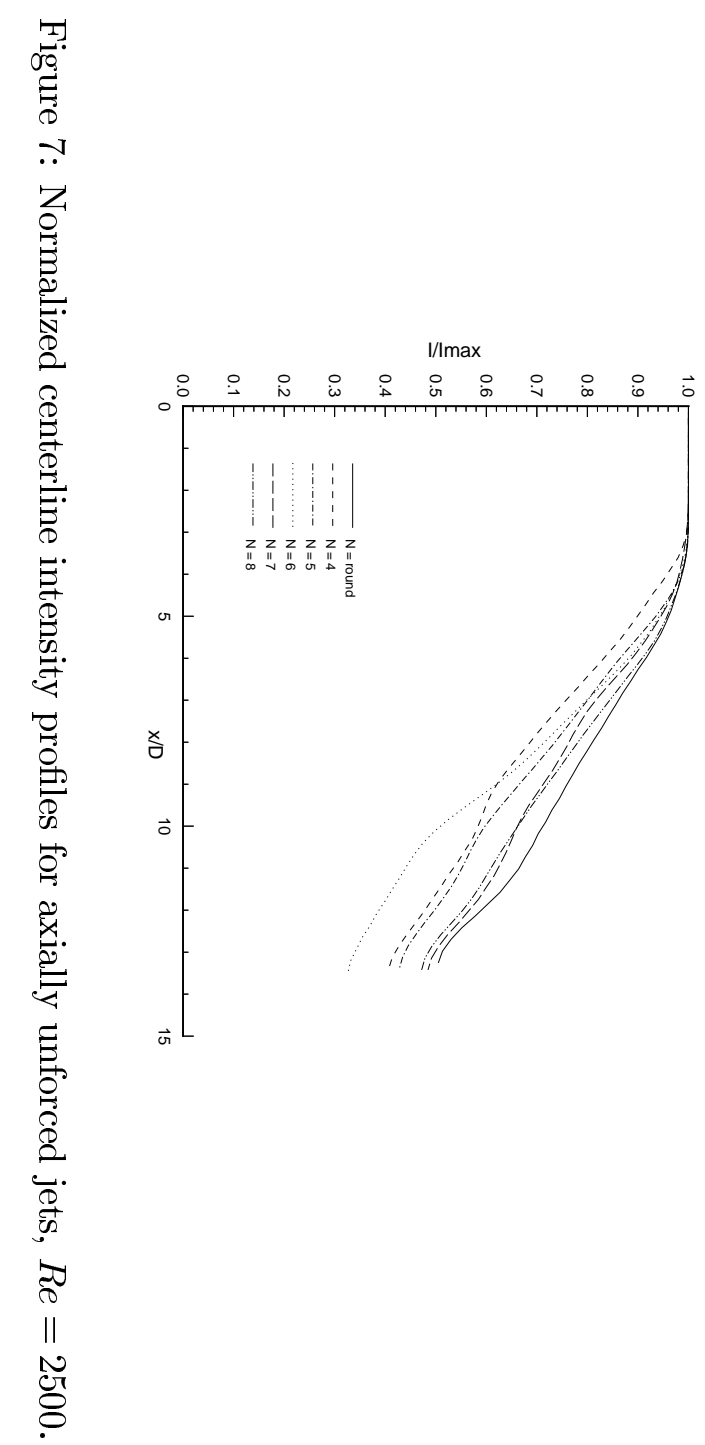




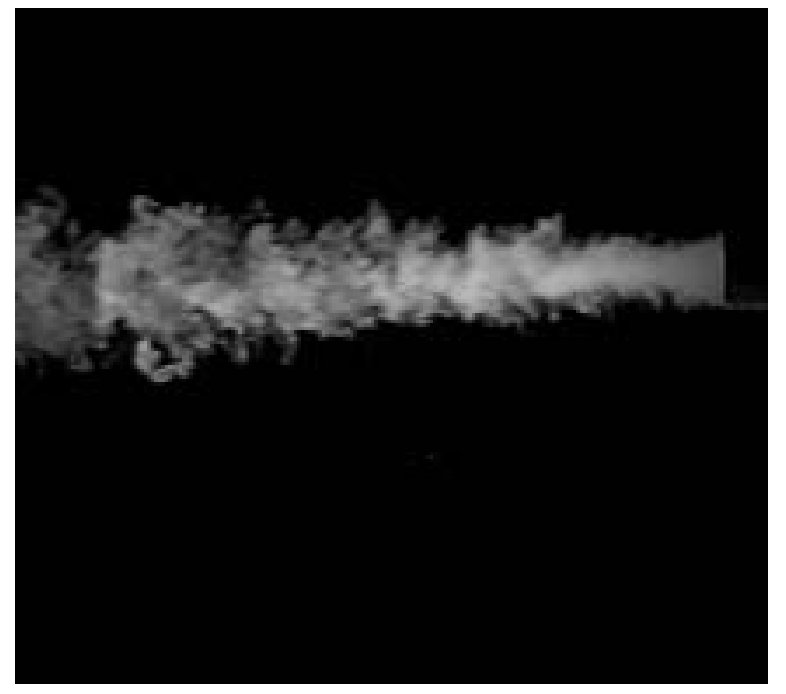

(a) $\mathrm{St}=0$

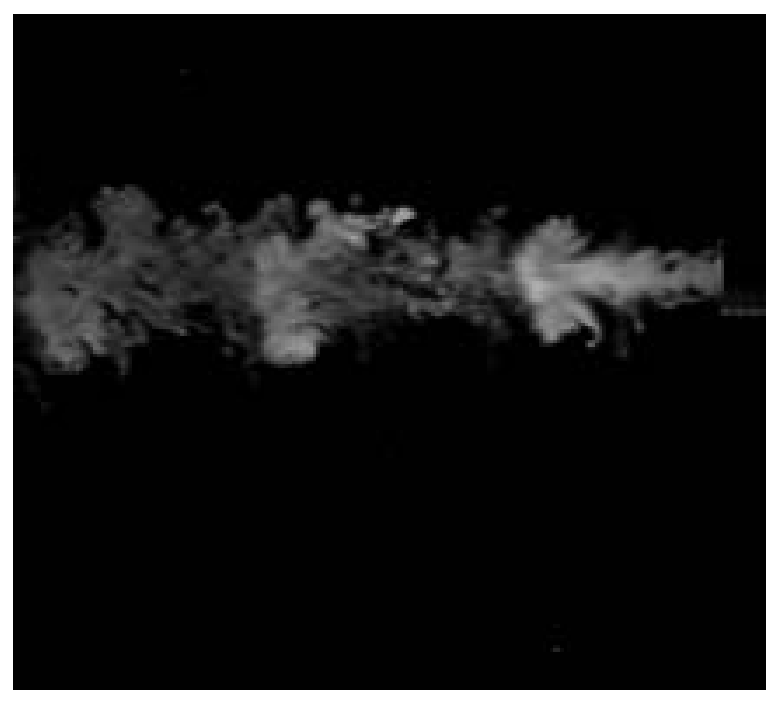

(c) $\mathrm{St}=0.67$

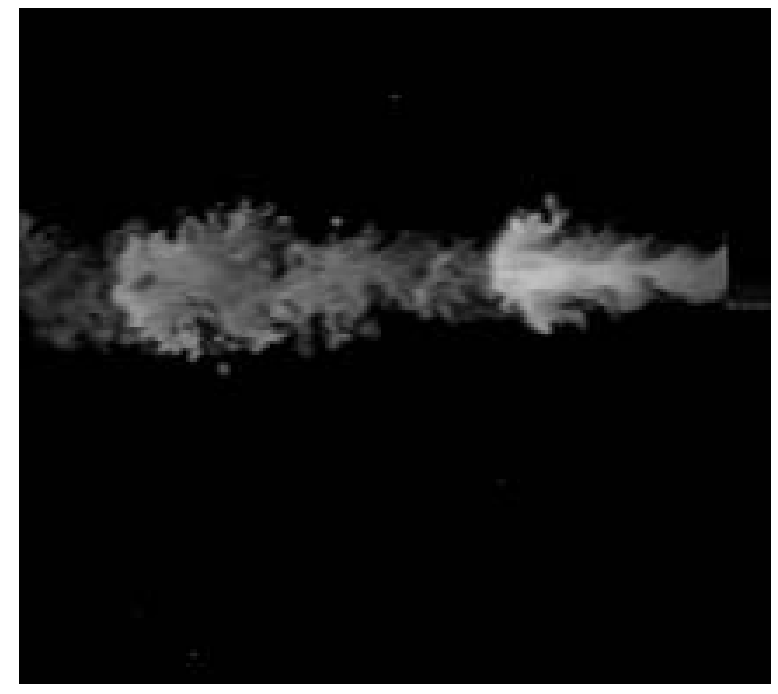

(b) $\mathrm{St}=0.47$

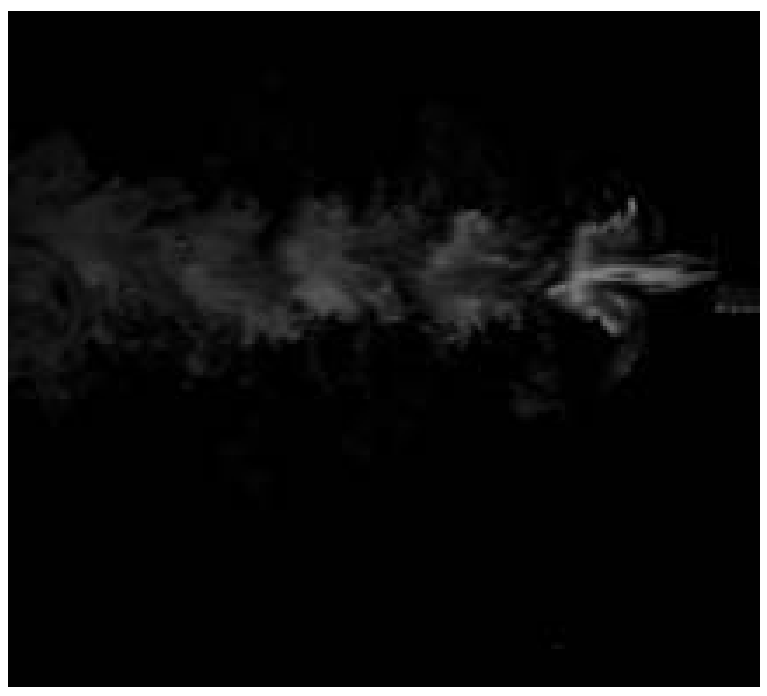

(d) $\mathrm{St}=1.3$

Figure 8: Instantaneous centerline images of 4-lobed jet at $R e=2000$. 

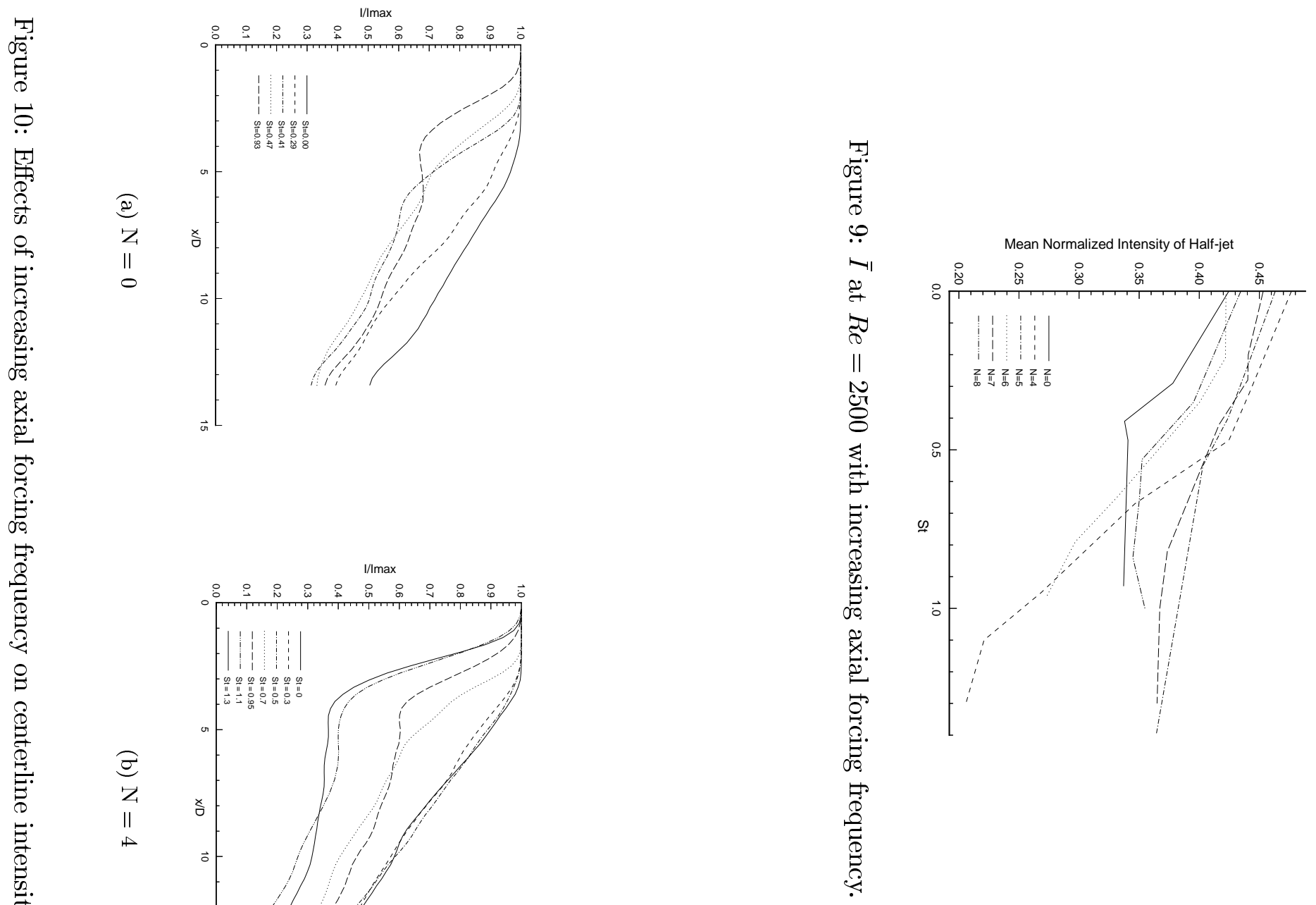

吾) 


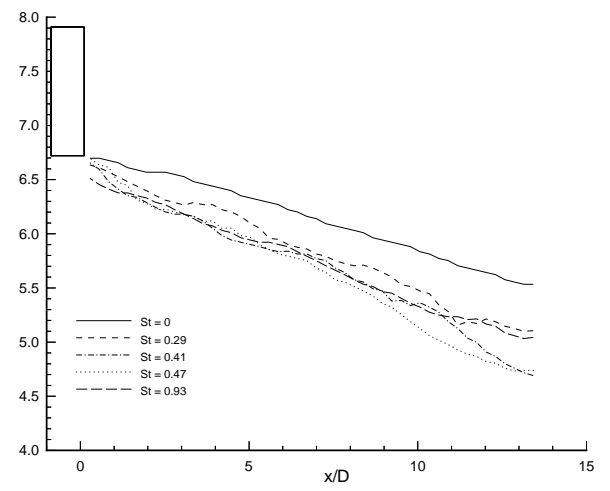

(a) $\mathrm{N}=0$

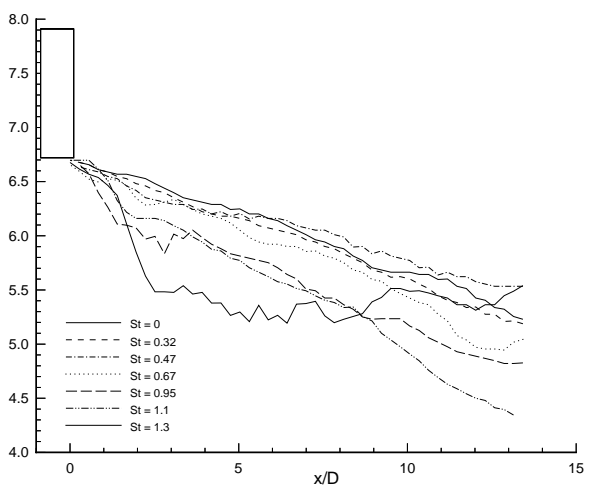

(b) $\mathrm{N}=4$

Figure 11: Effects of increasing axial forcing frequency on lateral spreading rates, $R e=2000$. 